\title{
Saturnian satellite observations made in Brazil during the 1995 opposition with an astrometric analysis
}

\author{
A. Vienne ${ }^{1,2}$, W. Thuillot ${ }^{1}$, C. H. Veiga ${ }^{3}$, J.-E. Arlot ${ }^{1}$, and R. Vieira Martins ${ }^{3}$ \\ 1 Institut de mécanique céleste et de calcul des éphémérides - Observatoire de Paris, UMR 8028 du CNRS, \\ 77 avenue Denfert-Rochereau, 75014 Paris, France \\ 2 Université de Lille, 9000 Lille, France \\ 3 Observatório Nacional, Rua Gal. José Cristino 77, 20921-400 Rio de Janeiro, Brazil
}

Received 7 May 2001 / Accepted 4 October 2001

\begin{abstract}
This paper provides an analysis of astrometric measurements of the main Saturnian satellites made thanks to CCD observations performed in 1995 at the Laboratório Nacional de Astrofísica at Itajubá in Brazil. The astrometric reduction is discussed especially the small corrections done here, but most of time neglected elsewhere. A catalog of 6006 differential positions has been obtained. They have been compared to different ephemerides, the Vienne \& Duriez ephemerides (TASS 1.7), the Harper \& Taylor ephemerides and the Dourneau ephemerides. These observations provide a large set of modern observations, and appear to be of good precision. This accuracy is needed for future use of these data to improve the dynamical models. These positions are included in the data base NSDC dedicated to the natural satellites (ftp://ftp.bdl.fr/pub/NSDC/saturn/raw_data/position/). They are also available at the CDS via anonymous ftp to cdsarc.u-strasbg.fr (130.79.128.5) or via http://cdsweb.u-strasbg.fr/cgi-bin/qcat?J/A+A/380/727
\end{abstract}

Key words. Saturn - satellites of Saturn - astrometry

\section{Introduction}

In 1995, an international campaign was organized in order to coordinate the observations of phenomena among the Saturnian satellite system (Arlot \& Thuillot 1993). During this period the Earth and Sun crossed the orbital planes of these satellites and mutual occultations and eclipses occurred. Many observers then planned to observe these events in order to catch the short light-flux drops most of times thanks to CCD cameras. Sixty-six such observations of events were successfully performed in several countries (Thuillot et al. 2001). But actually many CCD frames were then obtained without any detection of events because of the difficulty to measure a faint magnitude drop closely to the planet. Here we intend to extract the astrometric data from these observations in order to get a catalog of positions jointly with the observations of the events themselves.

These positions could subsequently be applied to the improvement of the dynamical models and to explore some specific problems. For example accurate positions of both Mimas and Tethys are necessary to well determine the eccentricity of Tethys which has a major influence on the evolution of the Mimas-Tethys resonance and lead to a

Send offprint requests to: A. Vienne, e-mail: Alain.Vienne@bdl.fr better knowledge of its dynamical history (Champenois \& Vienne 1999a, 1999b).

First we present a description of these measurements issued from the observations made at the Laboratório Nacional de Astrofísica at Itajubá in Brazil. We present our reduction, and after we show the comparison of the obtained positions with those deduced from the ephemerides. And at last, we give the catalog of the corresponding astrometric data. These data could also be accessed under a standard form on the data base NSDC dedicated to the natural satellites (ftp://ftp.bdl.fr/pub/NSDC/ saturn/raw_data/position/).

\section{The observations}

In Brazil, campaigns of astrometric observations of the natural satellites are performed (see for example Veiga \& Vieira Martins 1999). During the 1995 international campaign of observation of the Saturnian mutual events, several nights were scheduled at the Laboratório Nacional de Astrofísica at Itajubá to observe the Saturnian satellites. The number of observations and its distribution over the period 1995-1996 are given in Fig. 1.

The two telescopes used at Itajubá $\left(3^{\mathrm{h}} 2^{\mathrm{m}} 19^{\mathrm{s}}\right.$ East in longitude, $22^{\circ} 32^{\prime} 4^{\prime \prime}$ South in latitude, altitude $1872 \mathrm{~m}$ ) were the $1.60 \mathrm{~m}$ and the $0.60 \mathrm{~m}$ reflectors. They were 


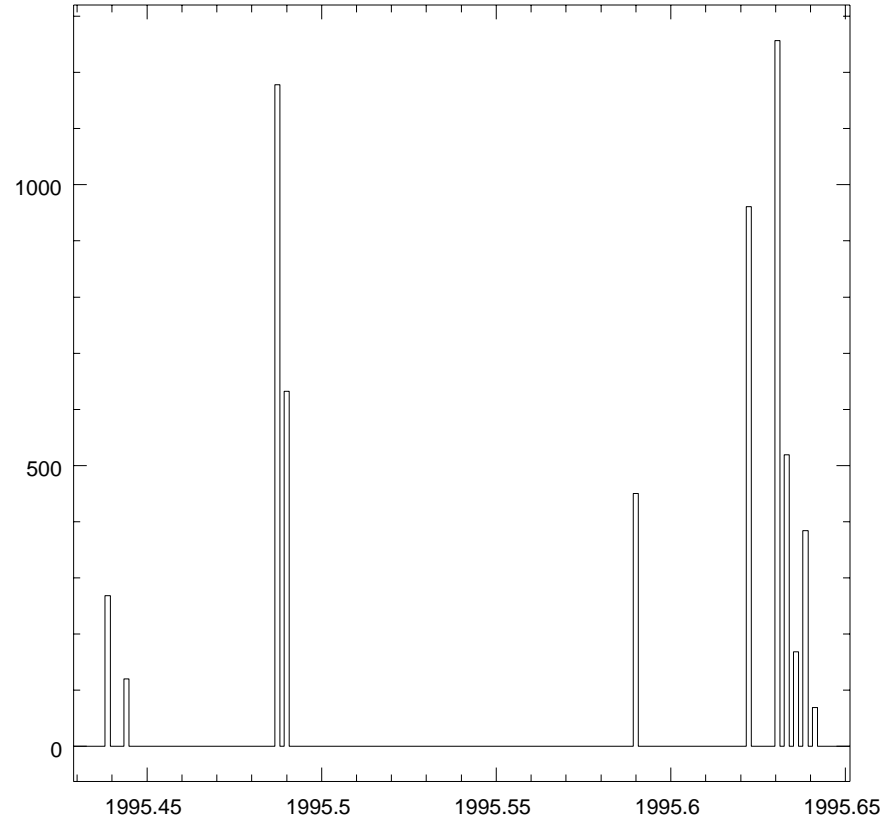

Fig. 1. Histogram of the observations with respect to times. Each bar corresponds to one night.

Table 1. Technical characteristics of the CCD cameras. The column "series" give the Code of series as given in Tables 4 and 5 .

\begin{tabular}{ccc}
\hline Camera & Description & series \\
\hline CCD0048 & EEV P88231 (770X1152) & 1 \\
CCD009 & EEV-05.20.0.202 (770X1200) & $2-3-4$ \\
CAM2 & SITe SI003AB (1024X1024) & 6 \\
\hline
\end{tabular}

equipped with CCD cameras the characteristics of which are given in Table 1.

All the frames were analyzed thanks to the ASTROL software (Colas 1996) which allowed us to use a centering algorithm based on the adjustment of a Point Spread Function (PSF).

No stars could generally be recorded, but in several frames some stars were although visible.

\section{The reduction}

\subsection{Frames with stars}

Several frames where stars are visible close to the satellites have been reduced thanks to the ESO software MIDAS (Warmels 1991) and a new algorithm for astrometric reduction named PRIAM recently developed by Fienga et al. (in preparation). This algorithm of reduction leads in these case to topocentric equatorial J2000 coordinates of the satellites and to the determination of the scale and orientation of the frames. Unfortunately, these frames are too few, so we have used these frames and the corresponding reductions only to validate our inter-satellite reduction.

\subsection{Inter-satellite reduction}

We give in this section the main points of the reduction, we can find in Vienne et al. (2001) more details and a discussion of the method for the case of inter-satellite measurements.

The frames registered and measured in pixels are not directly comparable with ephemeris, because they have been affected by some local effects. Before any comparison, it is necessary to take into account these effects due to refraction, stellar aberration, central projection (projection of the celestial sphere on the tangential plane of the focal point), light-travel time between satellites and topocentric parallax. These differential astrometric corrections are classical and we have made them in the present work. In the past, the precision level was about 0 ". 20 for inter-satellite astrometry. It is surely the reason that until now, concerning the inter-satellite observations of satellites of Saturn, all these astrometric corrections were not automatically taken into account by previous authors:

- Dourneau $(1993,1987)$ claimed that the stellar aberration "affects equally the positions of stars, Saturn and the satellites", and so, he ignores a correction up to 0. . 04 ;

- Harper \& Taylor (1994) claimed that the light time travel between satellites is negligible for inter-satellite reduction. And then, several authors neglect this effect in the case of the Saturnian system. This effect can reach 0 .'025 for Iapetus. In fact, the estimation of Harper \& Taylor was done neglecting the velocity of Saturn, and then leading to 0'!006 only for Iapetus;

- Harper et al. (1997) took into account the differential parallax, the aberration and the refraction (and not the others effects);

- The correction of the central projection is neglected by all the authors. But it can reach $0 . \prime 03$. Only Shen et al. (2001) do this correction. Nevertheless they suppose that the center of the frame is the reference satellite, so the correction falls down in most cases.

The precision level for inter-satellite astrometry is now about 0 .'05. Thus, these corrections have to be considered. Paradoxically, the topocentric parallax is taken into account by most of the observers, but, for inter-satellite measurements and for Saturn, the effect is very low ( $\leq 4$ mas).

The list of the differential corrections, to be applied to the five outer planetary sytems, is given in Table 2 . The values given in the right column are indicative; they correspond to a maximum value, for a differential effect applied to a standard frame $400^{\prime \prime}$ large. In the present set of observations, the geocentric elongation of Saturn from the Sun is greater than $85^{\circ}$, so the differential correction due to the relativistic deflexion of the Sun is less than 0.009 mas. Due to Saturn, this effect is about 0.02 mas in the position of Iapetus. So, in the present reduction, we have neglected these two effects.

The positions of Saturn are given by the ephemerides SLP96 from the "Institut de mécanique céleste 
Table 2. Differential effects in arcsec for a standard frame $400^{\prime \prime}$ large.

\begin{tabular}{ll}
\hline effect & correction \\
\hline refraction & $1^{\prime \prime}\left(z \leq 70^{\circ}\right)$ \\
stellar aberration & $0^{\prime \prime} 04$ \\
central projection (typical value) & $0^{\prime \prime} 03$ \\
light-travel time between satellites & $0^{\prime \prime} 001$ (Uranus) to $0^{\prime \prime} 044$ (Jupiter) \\
topocentric parallax & $0^{\prime \prime} 002$ \\
relativistic deflexion from the Sun & $0^{\prime \prime} 00014$ (geocentric elongation $\sim 20^{\circ}$ ) \\
relativistic deflexion from the planet & $0^{\prime \prime} 00005$ (Callisto), $0^{\prime \prime} 00002$ (Iapetus) \\
\hline
\end{tabular}

(IMCCE)" (available at ftp://ftp.bdl.fr/pub/ephem/ sun/slp96/) found on the VSOP87 planetary theory (Bretagnon \& Francou 1988). The precision on the positions of Saturn is about 0.' 4 which is enough here.

We use three different ephemerides for the saturnicentric positions of the satellites: TASS1.7 (Vienne \& Duriez 1995; Duriez \& Vienne 1997), H-T (Harper \& Taylor 1993) and Dourneau (1993). The time argument is the Terrestrial Time $(T T)$. At a given date corrected for the light-travel time between the satellites, we compute the apparent coordinates, $X_{\mathrm{c}}$ and $Y_{\mathrm{c}}$, of the objects. These coordinates take into account all the corrections listed above. We use the observed frame as a table of positions $\left(x_{\mathrm{p}}, y_{\mathrm{p}}\right)$, given in pixels (see Sect. 2). So, with the relation $\left(\begin{array}{rr}a & b \\ -b & a\end{array}\right)\left(\begin{array}{l}x_{\mathrm{p}} \\ y_{\mathrm{p}}\end{array}\right)-\left(\begin{array}{c}X_{\mathrm{c}} \\ Y_{\mathrm{c}}\end{array}\right)=0$, we compute an estimation $\widetilde{a}, \widetilde{b}$ of $a$ and $b$ by a least square procedure. We deduce the scale factor $\rho=\sqrt{\widetilde{a}^{2}+\widetilde{b}^{2}}$ and the orientation of the receptor (the angle from the $x_{\mathrm{p}}$-axis of the camera to the true equator) $\varphi=\arctan (\widetilde{b}, \widetilde{a})$. Note that, because of the linearity of the relation between $\left(X_{\mathrm{c}}, Y_{\mathrm{c}}\right)$ and $\left(x_{\mathrm{p}}, y_{\mathrm{p}}\right)$, no iterative procedure is necessary. It is not true for the direct determination of $\rho$ and $\varphi$ as it is done by Shen et al. (2001). Using $\rho$ and $\varphi$, and applying once more time, but inversely, the local corrections, we get the coordinates $(\alpha, \delta)$ of each measured object. The coordinates $(\alpha, \delta)$ are astrometric, but there is an absolute part $\alpha_{C}$ and $\delta_{C}$ which does not come from the observations. $C$ is defined such as the frame is the tangential plane of the celestial sphere at $C$. We have to know the position of this point in order to make the corrections of the central projection. As $C$ does not correspond to a physical object, we do not know $\left(\alpha_{C}, \delta_{C}\right)$. We estimate $\left(\alpha_{C}, \delta_{C}\right)$ by an iterative procedure beginning by applying the reduction without the present correction. Two iterations are enough to obtain a good estimation. In order to avoid to interpret the observations as absolute ones, we publish them in inter-satellite form $(\Delta \alpha \cos \delta, \Delta \delta)$, or more precisely, $\left(\alpha_{\mathrm{o}}-\alpha_{\mathrm{r}}\right) \cos \delta_{\mathrm{r}}$ and $\delta_{\mathrm{o}}-\delta_{\mathrm{r}}$. The index o is for the satellite object, and the index $r$ for the reference satellite. The content of the catalog is described in Sect. 5.

The procedure is applied once only for all frames of a given series. Generally, a series of frames corresponds to one campaign of observations and covers several nights. We then suppose that the receptor has been mounted in the same way for all the frames of the series.
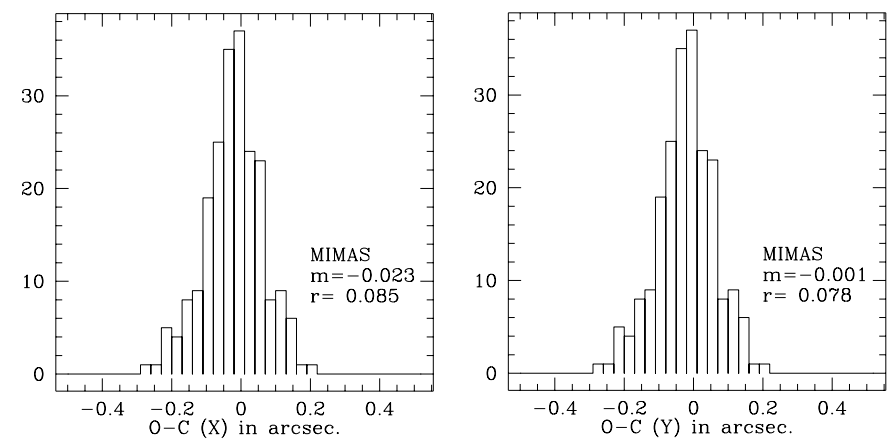

Fig. 2. Histogram for $\Delta \alpha \cos \delta(X)$ and $\Delta \delta(Y)$ residuals of Mimas relative to Rhea (if present).

This procedure is efficient for the eight major satellites of Saturn, or more generally for all objects for which we have ephemerides. But, in the least square procedure, only the positions of Tethys, Dione, Rhea and Titan are used to calibrate because these satellites have the best ephemerides, probably affected by the smallest systematic effects.

The positions $(\Delta \alpha \cos \delta, \Delta \delta)$ we have obtained are really astrometric ones because they are given in the J2000 system and all significant astrometric corrections have been done. But, for a given series, they are given apart from a scale factor and from a rotation. As explained in Vienne et al. (2001), if we want to compute the astrometric coordinates in any other way, for example with other ephemerides, we have only to touch up $\varphi$ and $\rho$.

\section{The comparison with ephemerides}

We use three different ephemerides for the saturnicentric positions of the satellites: TASS1.7, H-T and Dourneau. The position of Saturn itself is given by the ephemerides SLP96 (see above). The time argument is the Terrestrial Time $(T T)$.

The observations rejected are the same for the three theories: an observation is rejected when the residual is greater than $0 . .5$ for each of the three theories. In this way, we have rejected 6 observations of Mimas, 2 of Tethys, 1 of Titan and 12 of Hyperion.

The statistics of the comparison is shown in Table 3. Figures 2 to 8 show the corresponding histograms (for TASS only). From Table 3 and whatever the theory used, we can deduce that the dispersion of the observations 

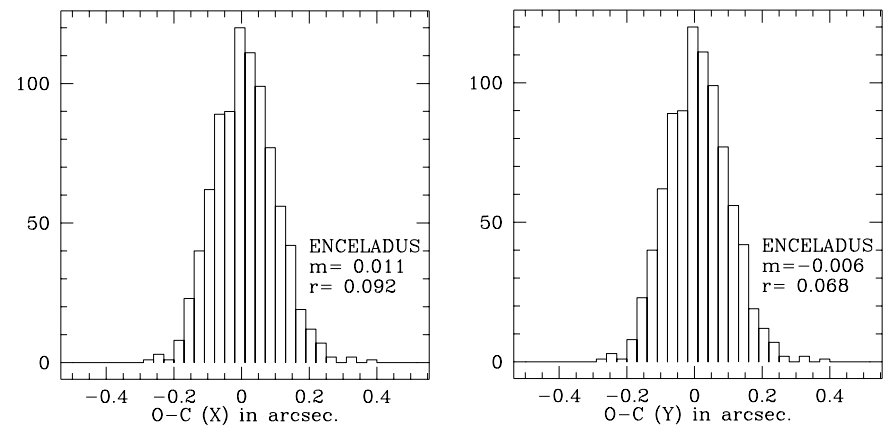

Fig. 3. Histogram for $\Delta \alpha \cos \delta(X)$ and $\Delta \delta(Y)$ residuals of Enceladus relative to Rhea (if present).
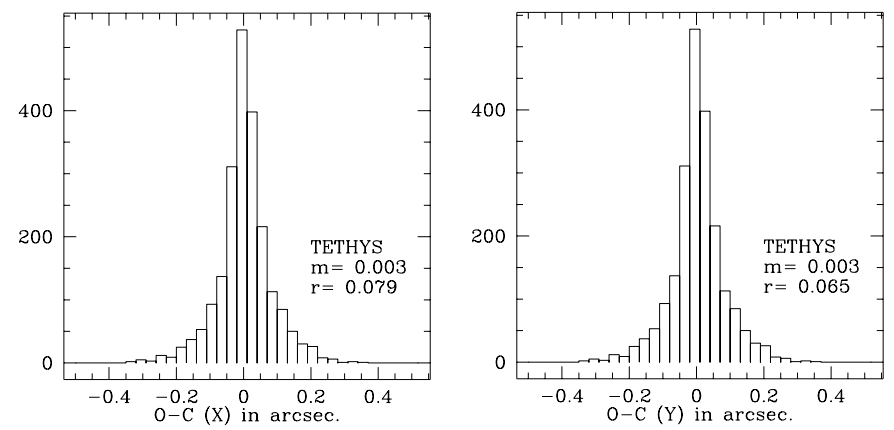

Fig. 4. Histogram for $\Delta \alpha \cos \delta(X)$ and $\Delta \delta(Y)$ residuals of Tethys relative to Rhea (if present).
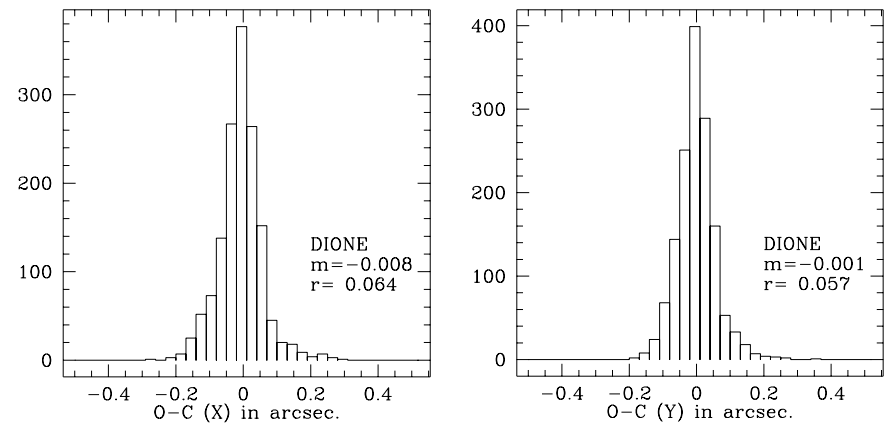

Fig. 5. Histogram for $\Delta \alpha \cos \delta(X)$ and $\Delta \delta(Y)$ residuals of Dione relative to Rhea (if present).

is about $0^{\prime \prime} 07$, and the bias of some milli-arcseconds. As Tethys, Dione, Rhea and Titan have been used in the fit, this fact is true for these satellites taken separately or globally. For Enceladus, the r.m.s of the residuals reaches $00^{\prime \prime} 08$, also whatever the used theory. It is not the case for Mimas, Hyperion and Iapetus. For these satellites, TASS gives the lowest residuals: 0".09 (Mimas), $00^{\prime \prime} 15$ (Hyperion), $00^{\prime \prime} 14$ (Iapetus) for $\Delta \alpha \cos \delta$, and $0^{\prime \prime} 08$ (Mimas), $0 ! 13$ (Hyperion), $0^{\prime \prime} 07$ (Iapetus) for $\Delta \delta$. Each one corresponds to several hundred positions: 216 for Mimas, 324 for Hyperion and 524 for Iapetus.

For comparison, Shen et al. (2001) have analyzed CCD observations over the 1990-1997 period from Qiao et al. (1999), Harper et al. (1997, 1999). From their analysis, they conclude that TASS is significantly more accurate $\left(0^{\prime \prime} 015\right)$ than other theories $\left(0{ }^{\prime \prime} 05\right)$. They give the following residuals: 0"'21 (Mimas), 0"16 (Hyperion), 0"'16 (Iapetus),
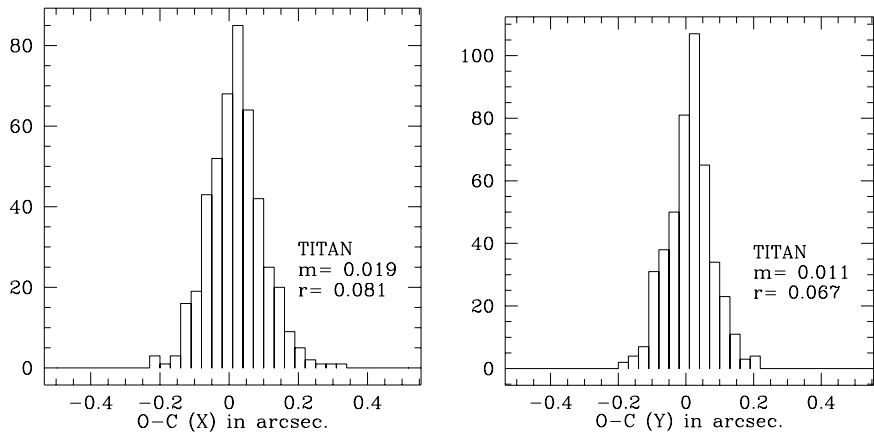

Fig. 6. Histogram for $\Delta \alpha \cos \delta(X)$ and $\Delta \delta(Y)$ residuals of Titan relative to Rhea.
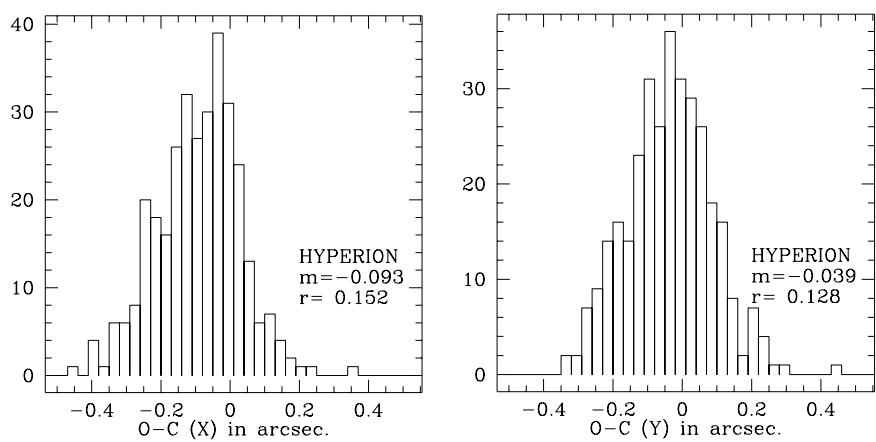

Fig. 7. Histogram for $\Delta \alpha \cos \delta(X)$ and $\Delta \delta(Y)$ residuals of Hyperion relative to Rhea (if present).
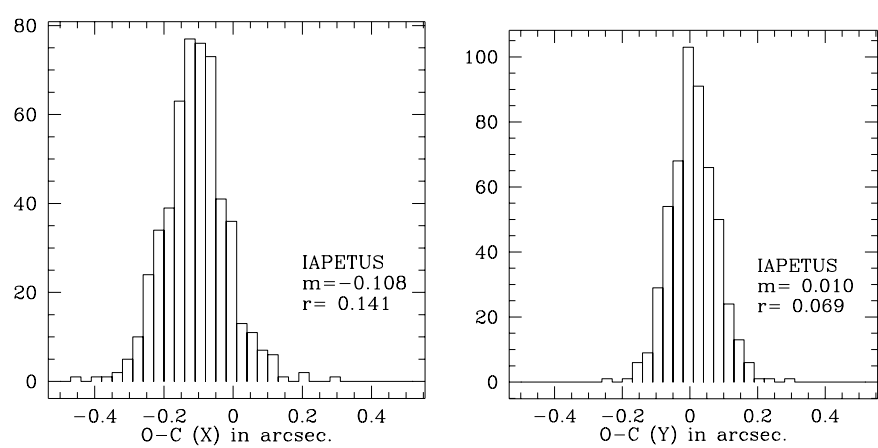

Fig. 8. Histogram for $\Delta \alpha \cos \delta(X)$ and $\Delta \delta(Y)$ residuals of Iapetus relative to Rhea (if present).

corresponding respectively to 57,218 and 230 positions. So, the observations presented in our paper cover a large set of modern observations, and are of good precision.

Many observations of Hyperion have bad residuals when the theory of Dourneau has been used. The reason is that Dourneau did not include any short period term in the expressions of Hyperion's theory. Note also, that Shen et al. (2001) use for the theory of Harper \& Taylor (H-T) a slight different version not yet published.

\section{The catalog of astrometric data}

The catalog is available in electronic form at ftp://ftp.bdl.fr/pub/NSDC/saturn/raw_data/position/. It is also available at the CDS via anonymous $\mathrm{ftp}$ to cdsarc.u-strasbg.fr $(130.79 .128 .5)$ or via http://cdsweb.u-strasbg.fr/cgi-bin/qcat?J/A+A/380/727. 
Table 3. Statistics of the $\mathrm{O}-\mathrm{C}$ (in mas) from the comparison with the ephemerides TASS, H-T and Dourneau. $N$ give the number of observations used. These residuals are relative to S5 (if S5 is absent of the frame, we use S6, and so on with the ordering list: S5, S6, S4, S3, S2, S1, S8 and S7). The last lines give the global residuals of S3, S4, S5 and S6 because the computed positions of these satellites have been used in the reduction.

\begin{tabular}{|c|c|c|c|c|c|}
\hline \multirow{2}{*}{$\begin{array}{c}\text { TASS } \\
\text { satellites }\end{array}$} & \multirow[b]{2}{*}{$N$} & \multicolumn{2}{|c|}{$\Delta \alpha \cos \delta$} & \multicolumn{2}{|c|}{$\Delta \delta$} \\
\hline & & means & $\mathrm{rms}$ & means & $\mathrm{rms}$ \\
\hline S1-Mimas & 216 & -23 & 85 & -1 & 78 \\
\hline S2-Enceladus & 865 & +11 & 92 & -6 & 68 \\
\hline S3-Tethys & 2151 & +3 & 79 & +3 & 65 \\
\hline S4-Dione & 1466 & -8 & 64 & -1 & 57 \\
\hline S6-Titan & 460 & +19 & 81 & +11 & 67 \\
\hline S7-Hyperion & 324 & -93 & 152 & -39 & 128 \\
\hline S8-Iapetus & 524 & -108 & 141 & +10 & 69 \\
\hline S3 S4 S5 S6 & 4077 & +1 & 74 & +2 & 62 \\
\hline H-T & & \multicolumn{2}{|c|}{$\Delta \alpha \cos \delta$} & \multicolumn{2}{|c|}{$\Delta \delta$} \\
\hline satellites & $N$ & means & $\mathrm{rms}$ & means & $\mathrm{rms}$ \\
\hline S1-Mimas & 216 & -54 & 108 & +12 & 80 \\
\hline S2-Enceladus & 865 & -3 & 90 & -10 & 69 \\
\hline S3-Tethys & 2151 & 0 & 79 & 0 & 65 \\
\hline S4-Dione & 1466 & -8 & 62 & +12 & 58 \\
\hline S6-Titan & 460 & 14 & 84 & +9 & 71 \\
\hline S7-Hyperion & 324 & -295 & 356 & +115 & 170 \\
\hline S8-Iapetus & 524 & +422 & 432 & +149 & 163 \\
\hline S3 S4 S5 S6 & 4077 & -2 & 74 & +5 & 63 \\
\hline \multirow{2}{*}{$\begin{array}{c}\text { Dourneau } \\
\text { satellites }\end{array}$} & & \multicolumn{2}{|c|}{$\Delta \alpha \cos \delta$} & \multicolumn{2}{|c|}{$\Delta \delta$} \\
\hline & $N$ & means & $\mathrm{rms}$ & means & $\mathrm{rms}$ \\
\hline S1-Mimas & 216 & -63 & 106 & +6 & 78 \\
\hline S2-Enceladus & 865 & -2 & 90 & -6 & 71 \\
\hline S3-Tethys & 2151 & +8 & 81 & +2 & 67 \\
\hline S4-Dione & 1466 & -1 & 64 & +8 & 59 \\
\hline S6-Titan & 460 & +3 & 89 & +8 & 74 \\
\hline (S7-Hyperion) & 324 & +1892 & 1924 & +98 & 167 \\
\hline S8-Iapetus & 524 & +206 & 225 & -34 & 78 \\
\hline S3 S4 S5 S6 & 4077 & +4 & 76 & +5 & 65 \\
\hline
\end{tabular}

It contains astrometric coordinates, in J2000 system, of the observations analyzed in Sect. 4 . Table 4 shows an extract of it. They are satellite/satellite positions expressed in arcsec, gathered by series with the corresponding scale (in pixels/arcsec) and orientation (in radians). The dates correspond to the mid-time of the pose. This date is not light-time corrected.

The format and the conventions of our catalog are very near to the one of Strugnell \& Taylor (1990) which contains most of the Earth-based observations. In a FORTRAN code, one line is read with the format: (i3, i5, i3, f11.7,f7.3, 2i4, i2, 1x , a2, a1, i2, i1, $2(1 \mathrm{x}, \mathrm{f} 13.7), 2 \mathrm{i} 2,2(1 \mathrm{x}, \mathrm{f} 7.3), 3 i 3,2 \mathrm{f} 10.3)$.
Table 4. Extract of the catalog.

\begin{tabular}{|c|c|c|}
\hline 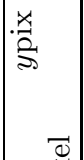 & 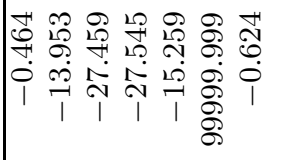 & 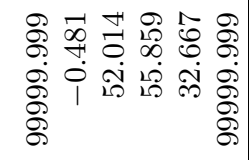 \\
\hline$\frac{x}{2}$ & 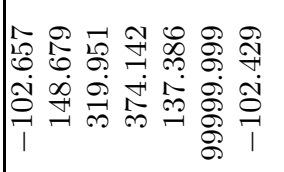 & 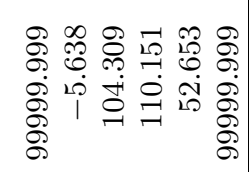 \\
\hline & 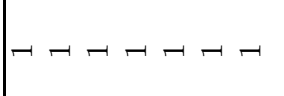 & 0000 \\
\hline & 10000000 & 0000000 \\
\hline & 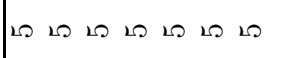 & 0000 \\
\hline 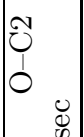 & 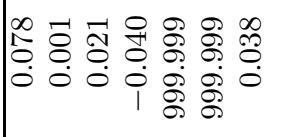 & 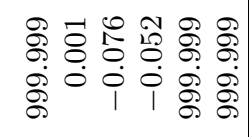 \\
\hline$\tau$ ت己 & 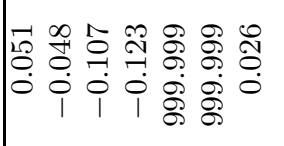 & 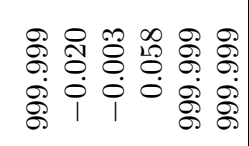 \\
\hline & $\neg \neg-\neg \neg-\neg$ & $\neg \neg-\neg$ \\
\hline & $\sim \sim \sim \sim \sim \sim \sim$ & $\sim \sim \sim \sim \sim \sim$ \\
\hline $\begin{array}{ll}0 & \\
0 & \\
0 & 0 \\
0 & 0\end{array}$ & 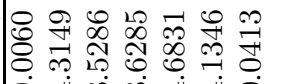 & 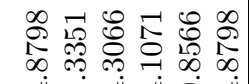 \\
\hline $\begin{array}{l}\overrightarrow{0} \\
\mathbb{8} \\
\stackrel{d}{5}\end{array}$ & 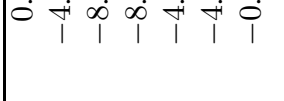 & 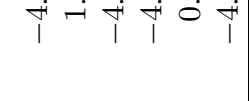 \\
\hline $\begin{array}{ll}0 & 0 \\
0 & 0 \\
0 & 0 \\
0 & 0\end{array}$ & 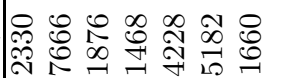 & 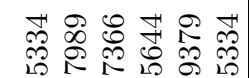 \\
\hline 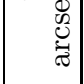 & 舟 & सं \\
\hline & 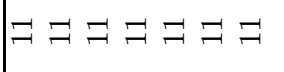 & 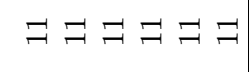 \\
\hline$\sigma$ & 象 & 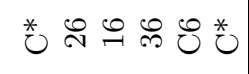 \\
\hline & $\neg-\neg-\neg 0-1$ & $0-r-r 0$ \\
\hline+ & 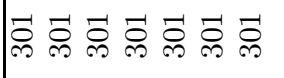 & 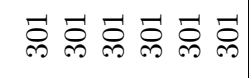 \\
\hline$\frac{n}{0}$ & $\underset{\infty}{\mathbb{N}} \underset{\infty}{\mathbb{N}} \underset{\infty}{\mathbb{N}} \underset{\infty}{\mathbb{N}} \underset{\infty}{\mathbb{N}} \underset{\infty}{\mathbb{N}} \underset{\infty}{\mathbb{N}}$ & $\underset{\infty}{\mathbb{N}} \underset{\infty}{\mathbb{N}} \underset{\infty}{\mathbb{N}} \underset{\infty}{\mathbb{N}} \underset{\infty}{\mathbb{N}} \underset{\infty}{\mathbb{N}}$ \\
\hline \begin{tabular}{ll}
$ن$ & $\infty$ \\
\multirow{2}{*}{} & $\infty$
\end{tabular} & 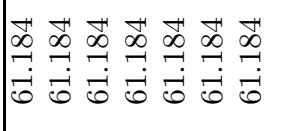 & 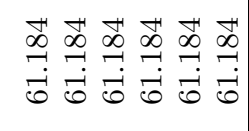 \\
\hline 0 & 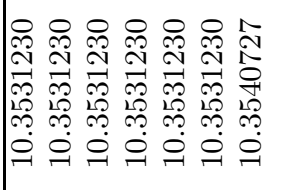 & 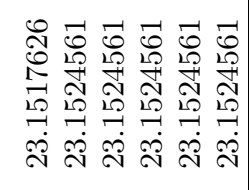 \\
\hline$\Xi$ & 0000000 & $\infty \infty \infty \infty \infty \infty$ \\
\hline 苂 & 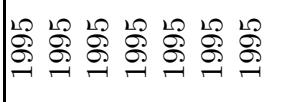 & : \\
\hline 8 & $\stackrel{\infty}{=}$ & $\stackrel{\infty}{=}$ \\
\hline
\end{tabular}


Table 5. Scale and orientation of each series of observations.

\begin{tabular}{ccc}
\hline series & $\begin{array}{c}\text { pixel } \\
(\operatorname{arcsec} / \mathrm{pix})\end{array}$ & $\begin{array}{c}\text { angle of orientation to equator } \\
(\mathrm{rad})\end{array}$ \\
\hline 1 & 0.294384053 & 0.004800195 \\
2 & 0.547902822 & 0.531254729 \\
3 & 0.547682483 & 2.625230525 \\
4 & 0.547603319 & 0.529115226 \\
6 & 0.547880584 & 0.530138505 \\
\hline
\end{tabular}

Mainly, we have changed the format in order to have more digits in the date, in the coordinates and in the $\mathrm{O}-\mathrm{C}$. In this way, the precision reaches the mas level. Of course, this precision is only the precision of the record.

The meanings of each parameter can be found in (Strugnell \& Taylor 1990), but we recall them below with eventually, in parenthesis, the differences with the original meaning:

- opp: opposition number, $1=1874$ to $118=1995$;

- year, m, utc: year, month and utc date of the observation (one more digit for utc: f11.7);

- tt-utc: TT-UTC in seconds (two more digits: f7.3);

- obs: UAI observatory code from the Minor Planet Center;

- ref: reference code of the catalog (we have chosen 301 for the present observations);

- t.: observation type. $0=\alpha, \delta, 1=\Delta \alpha \cos \delta, \Delta \delta, 2=$ $\Delta \alpha, \Delta \delta, 3=$ position angle, separation;

- obj.: subject satellite, reference satellite. 0 for Saturn, 1 to 8 for $\mathrm{S} 1$ to $\mathrm{S} 8$ (* when there is no reference satellite that is for observation type $0, \mathrm{C}$ for the center of the plate which is not a physical object);

- fl.: flags of presence of the first and second coordinates;

- obs1, obs2: first and second coordinates (one more digit, it is 0 when the coordinate is not present, see fl.);

- s: reference system. $0=$ mean equator and equinox B1950, 1 = true equator and equinox of date of observation, 2 = mean equator and equinox of J2000;

- f: reference frame. $0=$ topocentric, $1=$ geocentric, $2=$ heliocentric;

- $\mathrm{O}-\mathrm{C} 1, \mathrm{O}-\mathrm{C} 2$ : residuals of observations in arc-seconds (computed with TASS1.7 and with the format f7.3, it is 999.999 when the residuals are not computed and it is 888.888 when the residuals are greater than 100 . in absolute value);

$-(r$ : the number of the satellite used as reference in the computation of $\mathrm{O}-\mathrm{C})$;

- (-: no meaning in this series, index reserved);

- (s: the number of the series, the scale and orientation are the same for all the observations of one series);

- (xpix, ypix: original coordinates in pixels).

A priori, these two last columns are not necessary, but these data allow users of the catalog to adopt their own method of reduction. The coordinates are in arc-seconds for observations of type 1 , and in degrees for observations of type 0 . The other types are not used here.

For any catalog, it is important to know the method of reduction to allow future uses of the observations. It is why, we add at the end of our catalog, some lines which indicate how the reduction have been done and which corrections have been applied to get the coordinates. In particular, we give the scale and the orientation of each series of observations. These values are given in Table 5 . Note that the orientation refers to the true equator of the date because the calibration parameters are issued from a direct comparison between the observed frame and a "computed frame". But as explained in Vienne et al. (2001), it is possible to touch up the scale and the orientation, directly by comparing the observed astrometric coordinates and the computed ones (then given in the J2000 system). For example, a correction of these parameters could be useful to adjust dynamical theory. Another example, a new value for the mass of Saturn can be considered throughout the scale factor.

\section{Conclusion}

We give 6006 differential positions of the main Saturnian satellites issued from astrometric measurements of CCD observations performed in 1995 at the Laboratório Nacional de Astrofísica at Itajubá in Brazil. Most of these frames have no reference stars, then we applied an intersatellite reduction.

We have used the positions of Tethys, Dione, Rhea and Titan given by TASS1.7 to determine the scale factor and the orientation of the receptor.

The comparison of the observed positions and the computed positions shows that the dispersion of the observations is 0 .'07. Furthermore, we point out the quality and the number of the positions of the satellites not used in the calibration: $0 . \prime 08$ for the 216 positions of Mimas, 0 .' 14 for Hyperion (324), 0 '! 11 for Iapetus (524).

The catalog of the corresponding astrometric data could also be accessed under a standard form on the data base NSDC dedicated to the natural satellites (ftp://ftp.bdl.fr/pub/NSDC/saturn/raw_data/position/). The format and the conventions of our catalog are very near to the one of (Strugnell \& Taylor 1990). The coordinates are given apart from a scale factor and from a rotation, but all astrometric corrections are done. So, these differential positions are really astrometric ones in that meaning that, no astrometric consideration is necessary to use them, even if one wants to touch up the calibration. Nevertheless, the raw pixels are also given in order to allow anyone to reduce again the frames.

Acknowledgements. We thank all the colleagues and students who joined the campaign of observation of the Saturnian satellites at Itajubá, especially Marcelo Assafin, Serguei Puliaev, Marildo G. Pereira and Daniela Lazzaro. 


\section{References}

Arlot, J.-E., \& Thuillot, W. 1993, Icarus, 105, 427 Bretagnon, P., \& Francou, G. 1988, A\&A, 202, 309 Champenois, S., \& Vienne, A. 1999a, Celest. Mech. Dynam. Astrom., 74, 111

Champenois, S., \& Vienne, A. 1999b, Icarus, 140, 106

Colas, F. 1996, in Proc. of the PHESAT95 workshop, Supp. Ann. de Phys., 21, 133

Dourneau, G. 1987, Ph.D. Thesis, Bordeaux University

Dourneau, G. 1993, A\&A, 267, 292

Duriez, L., \& Vienne, A. 1997, A\&A, 324, 366

Harper, D., \& Taylor, D. B. 1993, A\&A, 268, 326

Harper, D., \& Taylor, D. B. 1994, A\&A, 284, 619

Harper, D., Murray, C. D., Beurle, K., et al. 1997, A\&AS, 121,65

Harper, D., Beurle, K., Williams, I. P., et al. 1999, A\&AS, 136, 257
Qiao, R. C., Shen, K. X., Liu, J. R., \& Harper, D. 1999, A\&AS, 137,1

Shen, K. X., Dourneau, G., Qiao, R. C., \& Liu, J. R. 2001, A\&A, 367, 1061

Strugnell, P. R., \& Taylor, D. B. 1990, A\&AS, 83, 289

Thuillot, W., Arlot, J. E., Ruatti, C., et al. 2001, A\&A, 371, 343

Veiga, C. H., \& Vieira Martins, R. 1999, A\&AS, 139, 305

Vienne, A., \& Duriez, L. 1995, A\&A, 297, 588

Vienne, A., Thuillot, W., \& Arlot, J. E. 2001, Astrometric reduction of CCD observations of planetary satellites without reference star, in Note Sci. et Tech. de l'I.M.C.C.E., S077

Warmels, R. H. 1991, The ESO-MIDAS System, in Astronomical Data Analysis Software and Systems I, PASP Conf. Ser., 25, 115 